\begin{tabular}{|c|c|}
\hline Title & $\begin{array}{l}\text { A Novel A ssessment Methodology for the EMI Occurrence in Implantable Medical Devices Based U pon Magnetic Flux } \\
\text { Distribution of RFID Reader/writers }\end{array}$ \\
\hline Author(s) & Futatsumori, Shunichi; Hikage, Takashi; Nojima, Toshio; Koike, Ben; Fujimoto, Hiroshi; Toyoshima, Takeshi \\
\hline Citation & $\begin{array}{l}\text { ISBN: 1-4244-1350-8, 1-6 } \\
\text { https://doi.org/10.1109/SEMC.2007.65 }\end{array}$ \\
\hline Issue Date & 2007-07-09 \\
\hline Doc URL & http:/hdl.handle.net/2115/38993 \\
\hline Rights & $\begin{array}{l}\text { (C)2007 IEEE. Personal use of this material is permitted. However, permission to reprint/republish this material for } \\
\text { advertising or promotional purposes or for creating new collective works for resale or redistribution to servers or lists, } \\
\text { or to reuse any copyrighted component of this work in other works must be obtained from the IEEE. }\end{array}$ \\
\hline Type & proceedings \\
\hline Note & EMC 2007 (IEEE International Symposium on Electromagnetic Compatibility, 2007). 9-13 July 2007. Honolulu, HI \\
\hline File Information & EMC2007_p1-6.pdf \\
\hline
\end{tabular}

Instructions for use 


\title{
A Novel Assessment Methodology for the EMI Occurrence in Implantable Medical Devices Based Upon Magnetic Flux Distribution of RFID reader/writers
}

\author{
Shunichi Futatsumori, Takashi Hikage, Toshio Nojima \\ Graduate School of Information Science and Technology \\ Hokkaido University \\ Sapporo, Japan \\ futatsumori@emwtinfo.ice.eng.hokudai.ac.jp \\ \{hikage,nojima\}@wtemc.ist.hokudai.ac.jp
}

\author{
Ben Koike \\ Japan Automatic Identification Systems Association \\ Tokyo, Japan \\ benkoike@jaisa.or.jp
}

\author{
Hiroshi Fujimoto, Takeshi Toyoshima \\ Medtronic Japan Co., Ltd. \\ Tokyo, Japan \\ \{hiro.fujimoto, takeshi.toyoshima\}@medtronic.com
}

\begin{abstract}
With respect to the EMI affecting implantable medical devices caused by Radio Frequency Identification Reader/Writers (RFID R/W), experimental investigations are conducted to develop a risk assessment methodology based upon the measurement of the EMF distribution around RFID R/W. First, fundamental EMI occurrence mechanisms of implantablecardiac pacemakers and -cardioverter-defibrillators (ICD) are described. Secondly, the characteristics of the radio wave emitted by the RFID R/W are described. Next, EMI test experiments and measurements of the field distributions are performed. Then, a novel experimental assessment methodology is proposed. The EMI characteristics derived from magnetic flux distributions are compared with the experimental results to confirm the effectiveness of the proposed methodology. Finally, it is shown that the probability of EMI occurrence can be estimated using the statistical processing of the proposed methodology.
\end{abstract}

Keywords-component; assessment methodlogy; electoromagnetic interference; implantable cardiac pacemaker; implantable cardioverter-defibrillator; Radio Frequency Identification;

\section{INTRODUCTION}

Wireless communication devices such as mobile phones, Radio Frequency Identification (RFID), Electric Article Surveillance (EAS), and contact less IC cards would be essential components to make up the ubiquitous society. However, radio wave from these devices has an incidental possibly to affect other electronics devices and cause unwanted malfunctions in certain circumstances. Accordingly, investigations of electromagnetic interference (EMI) have more importance these days. In particular, EMI effects on medical equipment, including implantable medical devices, such as implantable-cardiac pacemakers and -cardioverter-defibrillators (ICD) should be precisely investigated because these are used widely in general circumstances. A lot of research is being carried out to find out these effects [1] - [7]. In Japan, some guidelines to prevent such EMI is provided and operated based on this research [8], [9].

Among all of these wireless communication devices, RFID technologies are expected to achieve various applications and are expanding practical applications to various living spaces. The guidelines announced in Japan recently are derived from the report [9]. We have also carried out detailed EMI test experiments [10], [11] as an independent project. The final goal of this research project is development of an EMI assessment methodology using numerical analysis. In addition, this project includes investigations for providing technical information to create RFID reader/writers with less effect on implantable medical devices.

For low-band RFID reader/writers using $125 \mathrm{kHz}$ and HF (13.56 MHz), an inductive coupling between reader/writers and tags allows communications with each other. An experimental assessment on pacemakers and ICDs EMI based on magnetic flux distributions from their antennas are conducted. It is very important to confirm the estimation methodology for EMI using electromagnetic field (EMF) measurement results, because it is an essential element of the EMI assessment methodology using numerical analysis. To apply this methodology for EMF strength obtained by the computer simulation, the interference can be estimated using analysis results. Furthermore, technical information to create RFID reader/writers with less EMI characteristics will be provided to their manufactures when the threshold EMF levels or the EMI 
characteristics depending on 3-dimensional EMF distributions are obtained.

In this paper, a novel experimental assessment methodology for pacemakers and ICDs EMI due to magnetic flux distributions from low-band RFID reader/writer are discussed. First, the EMI occurrence mechanism of pacemakers and ICDs are described. An EMI characteristic due to alternating magnetic field is explained as well. Secondly, the characteristics of the radio wave emitted by the RFID R/W are described. Next, EMI test experiments and measurements of the field distributions are described. Then, novel experimental assessment methodology is proposed. EMI characteristics derived from magnetic flux distributions are compared with the experimental results to confirm the effectiveness of the proposed methodology. Finally, it is shown that the probability of EMI occurrence can be estimated using the statistical processing which is the result of the proposed methodology.

\section{EMI OCCURRENCE MECHANISM}

EMI on pacemakers and ICDs occurs when the following two situations are satisfied. First, the sensing circuit of pacemakers and ICDs receives a signal similar to an electrocardiogram (ECG) signal or receives obvious noise. Then, the disturbing signals' strength must be higher than the sensing threshold level. There are 3 factors which cause this condition - directly conducted current, alternating magnetic field and high voltage electric field [3]. EMI due to low-band RFID reader/writers is assumed to be caused by alternating magnetic field from antennas. In this case, it is supposed that "one turn coil" is connected between different electrode and indifferent electrode of pacemakers and ICDs, since the tissues of the human body are electric conductors. The magnetic flux density through the coil varies when the alternating magnetic field is generated from RFID reader/writer antennas. Then, by following Faraday's law of induction, an electromotive force arises between the electrodes. If this electromotive force exceeds the pacemakers' sensing threshold level, they cannot detect ECG signal any more. This is the mechanism of EMI due to alternating magnetic field.

Some features of pacemakers' two electrodes are explained. To sense/pace ECG signal, they have two electrodes - a different electrode and an indifferent electrode. There are two pacemaker operating modes depending on the position of the electrodes. One mode is called "unipolar mode". A metal housing of pacemakers and a tip electrode of lead wire carries a sensing/pacing signal. Another is called "bipolar mode". A tip electrode and a ring electrode both located at the end of a coaxial lead wire carry a sensing/pacing signal. When the EMI is caused by an alternating magnetic field, bipolar mode enables us to reduce the effects by $1 / 6$ to $1 / 10$ compared with unipolar mode.

Pacemakers and ICDs have mechanisms to prevent EMI such as a noise reversion mode, sensing filter and adjustable sensing threshold level. These mechanisms protect it from the effects as much as possible. However, it is difficult to prevent all effects by only using these functions. Especially for the disturbance noise has the same period as ECG signal, these EMI protection mechanisms may lose their functions.

\section{SPECIFICATIONS FOR LOW-BAND RFID READER/WRITERS}

RFID reader/writers using $125 \mathrm{kHz}$ and the HF band have possibilities to generate alternating magnetic fields described above. Therefore, these low-band RFID systems employ inductive coupling for communication method with tags. Backscatters of magnetic filed reflected by the tags enable data transmission.

$125 \mathrm{kHz}$ RFID systems are used for maintenance of facilities and restaurants. In addition, HF band RFID systems are the most widely used system around the world. Applications of this frequency band RFID system include supply chain management, passengers' baggage management in the airlines and contact less IC cards which is used for automatic ticket checker in the station. Typical characteristics of each frequency band are shown in Table I. International standard ISO/IEC $18000-2$ for $125 \mathrm{kHz}$ and ISO/IEC 18000-3 for HF band were published on June 2004 as specifications for air interface. ISO/IEC 15693, which is used as specifications for contact less IC card, is almost same as ISO/IEC 18000-3 mode-1. These RFID systems are required to fulfill the regulations for radio wave and electromagnetic compatibility (EMC) operating by the government of the country or community as well.

TABLE I. TYPICAL CHARACTERISTICS OF LOW-BAND RFID SYSTEM

\begin{tabular}{|c|c|c|}
\hline Frequency bands & $\begin{array}{c}125 \mathrm{kHz}(135 \mathrm{kHz} \text { or } \\
\text { lower })\end{array}$ & $\mathrm{HF}(13.56 \mathrm{MHz})$ \\
\hline Transmission method & Magnetic coupling & Magnetic coupling \\
\hline $\begin{array}{c}\text { Communication } \\
\text { distance }\end{array}$ & $0-20 \mathrm{~cm}$ & $0-60 \mathrm{~cm}$ \\
\hline Specifications & ISO/IEC $18000-2$ & $\begin{array}{c}\text { ISO/IEC } 18000-3 \\
\text { ISO/IEC } 15693\end{array}$ \\
\hline Modulation method & ASK & ASK \\
\hline
\end{tabular}

TABLE II. Tested IMPlantable MedicAl Devices

\begin{tabular}{|c|c|c|}
\hline Tested devices & Type of chambers & Number of devices \\
\hline \multirow{2}{*}{ Pacemaker } & Single chamber & 5 \\
\cline { 2 - 3 } & Dual chamber & 5 \\
\hline Implantable & Single chamber & 1 \\
\cline { 2 - 3 } Cardioverter-Defibrillator & Dual chamber & 2 \\
\hline Total & & 13 \\
\hline
\end{tabular}

TABLE III. RFID READER/WRITER ANTENNAS

\begin{tabular}{|c|c|c|}
\hline Frequency bands & $125 \mathrm{kHz}$ & HF $(13.56 \mathrm{MHz})$ \\
\hline Specifications & - & ISO/IEC 15693 \\
\hline Modulation method & ASK & ASK \\
\hline Number of antennas & 4 & 2 \\
\hline
\end{tabular}

\section{EMI TEST EXPERIMENTS AND FIELD DISTRIBUTION MEASUREMENTS}

In this section, the EMI test experiments and the measurement of field distribution from low-band RFID reader/writer antennas are described [10], [11]. First, in vitro experiments for the assessment of EMI due to RFID reader/writer are explained. Then, measurements of field distributions from low-band RFID reader/writer antennas are introduced. 


\section{A. EMI test experiments}

EMI test experiments for 10 types of antennas and 13 types of pacemakers and ICDs have been carried out so far. Detailed EMI characteristics such as the levels of interference and the maximum interference distance (distance where EMI disappears) are obtained. EMI due to 4 types of $125 \mathrm{kHz}$ antennas and 2 types of HF band antennas are examined. Tested pacemakers and antennas are shown in Table II and Table III. Radio waves from all RFID reader/writer antennas used in the experiments are not on and off output signal but continuous signal output employing modulation method as amplitude-shift keying (ASK).

The test system is based upon the previously proposed one for the estimation of EMI due to mobile phones described in [1], [2], [4] and [12]. The system is also employed in EMI test experiments reported by the Ministry of Internal Affairs and Communications (MIC) of Japan [7]. As shown in Fig. 1, the test system consists of a flat human torso phantom, an ECG signal generator/detector, a chart recorder, an oscilloscope, a measurement platform and a RFID reader/writer antenna.

The important part of the EMI assessment based on magnetic flux distributions is the human torso phantom applied for the EMI test experiments. As shown in Fig. 2, the torso phantom is a modification of Irnich's model as described in the reference [10], [11]. A key feature of this phantom is that it is designed to have a higher induced voltage than that of actual operating situations implanted in human bodies. Induced voltage is caused by the external magnetic field. The "one turn coil" which is surrounded by the lead wire and two electrodes is shown in Fig. 3. When the EMI is caused by alternating magnetic filed, the strength of the noise is proportion to the magnetic flux which is through cross-section area of $S$. Electrode of this phantom is placed on the half round shape, to obtain a maximum cross-section area $S$ of $573 \mathrm{~cm}^{2}$. It is reported that this value can give twice as much conservative results for $98 \%$ of pacemakers and ICDs users, by comparing with the actual cross-section area of human bodies [3], [7].

The operating modes of pacemakers and ICDs are VVI mode (the Ventricle chamber is paced, the Ventricle chamber is sensed, and the response to sensing is Inhibited) and AAI mode (the Atrium chamber is paced, the Atrium chamber is sensed, and the response to sensing is Inhibited). For each of the operating modes, two types of EMI test modes are conducted one with an injected ECG signal (typical inhibition), and one with no injected ECG signal (typical asynchronous pacing). The Procedure of the EMI test procedure is shown in follows.

1) Program both sensitivity and refractory period of pacemakers and ICDs to maximum sensitivity and minimum time, respectively. This setting gives the most conservative results.

2) Set configurations of RFID reader/writers to actual operating mode.

3) Move antennas from side to side in the $y$-axis direction in Fig. 1 while doing the experiments. This generates changing magnetic fields. The velocity of this movement is 1 $\mathrm{m} / \mathrm{s}$, similar to the speed of walking.
4) Record ECG signal for each mode on the paper for 100 seconds. The distance between antennas and the torso phantom ( $x$-axis direction in Fig. 1) is increased when interference occurs. In this case, the maximum interference distance (distance where EMI disappears) is determined and recorded in centimeters.

5) Step down sensitivity of pacemakers and ICDs to five levels (maximum, $1.0 \mathrm{mV}, 2.4 \mathrm{mV}, 5.6 \mathrm{mV}$, and minimum) and record the maximum interference distance, as long as interference exists.

6) Carry out experiments for combinations of all antennas and implantable devices. The operating modes of pacemakers and ICDs include unipolar/bipolar mode and VVI/AAI mode.

The total of 480 test modes are conducted using the test procedure described above.

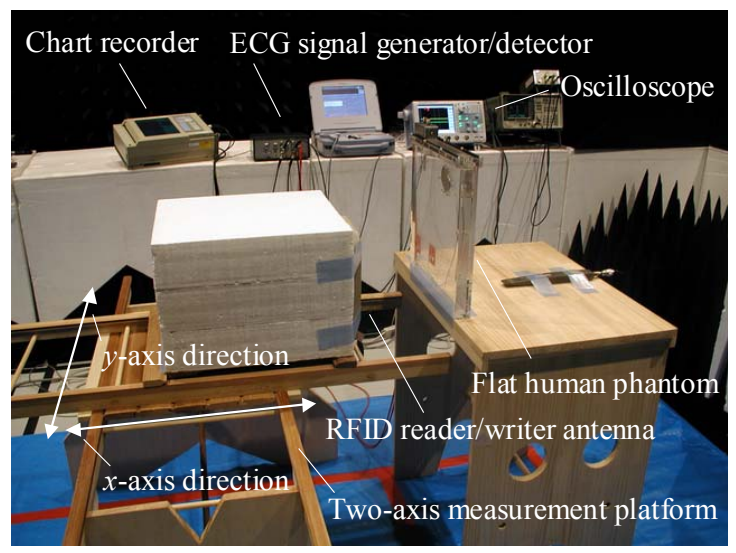

Figure 1. EMI test experimental system.

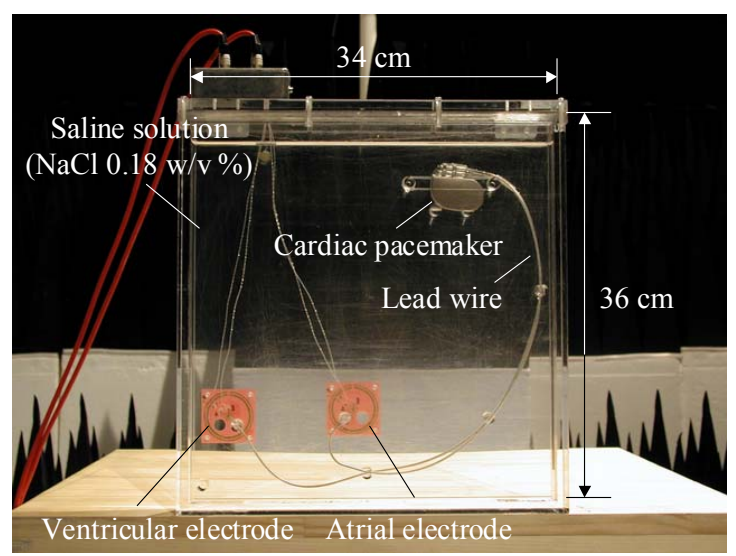

Figure 2. The human torso phantom. 


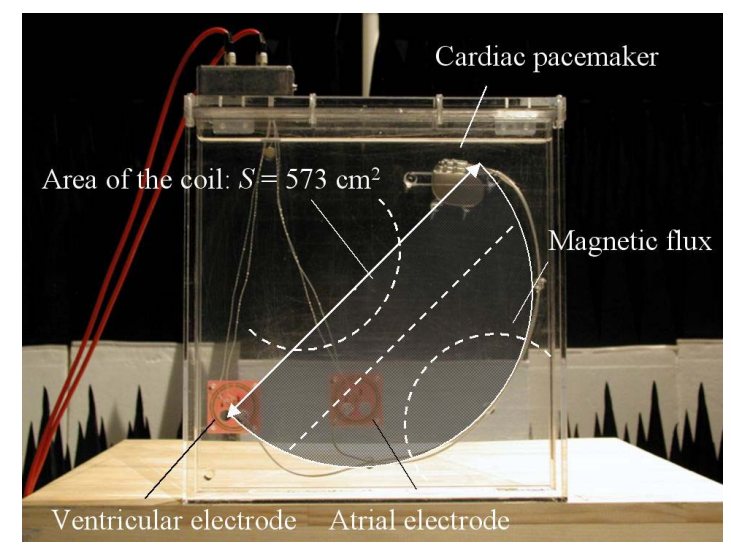

Figure 3. Area of the "one turn coil" consists of the lead wire and pacemkaers.

\section{B. Measurement of field distribution}

To assess pacemakers and ICDs EMI based on magnetic flux distributions from low-band RFID reader/writers, detailed EMF distributions from antennas are measured. Both electric and magnetic field strength are determined for all RFID reader/writers antennas shown in Table III.

A total of 729 sample points including $70 \mathrm{~cm} \times 70 \mathrm{~cm} \times 70$ $\mathrm{cm}$ (width $\times$ height $\times$ depth) with $10 \mathrm{~cm}$ separation are measured. In addition to this wide area measurement, we examined a finer separation for the depth direction ( $x$-axis direction in Fig. 1) based on the maximum interference distance obtained from the EMI test experiments. A total of 1029 sample points including $70 \mathrm{~cm} \times 70 \mathrm{~cm} \times 20 \mathrm{~cm}$ (width $\times$ height $\times$ depth) at $1 \mathrm{~cm}$ separation for the depth direction are measured.

Only magnetic field strength is employed for the assessment, because the electric field from low-band RFID reader/writer antennas is much smaller than the magnetic field and negligible for EMI occurrence. Fig. 4 and Fig. 5 shows examples of magnetic field distributions from 2 types of $\mathrm{HF}$ band antennas. Both distributions are magnetic field strength from the surface of each antenna $(x=0 \mathrm{~cm})$. The coordinate origin is the central axis of the antennas. In addition, values of $y$-axis are normalized to the maximum magnetic field strength out of 2 types of antennas.

\section{EMI ASSESSMENT BASED ON MAgNetic FluX DISTRIBUTIONS}

\section{A. An experimental assessment methodology}

In this section, a novel experimental assessment methodology for implantable medical devices EMI based on magnetic flux distribution is proposed. In addition, EMI characteristics assessed by magnetic flux distributions are compared with those obtained by the experiments. This assessment methodology is conducted and the procedure is as follows.

1) Assume that the central axis of RFID reader/writer antennas are positioned where maximum interference is detected at the EMI test experiments. Then, calculate the

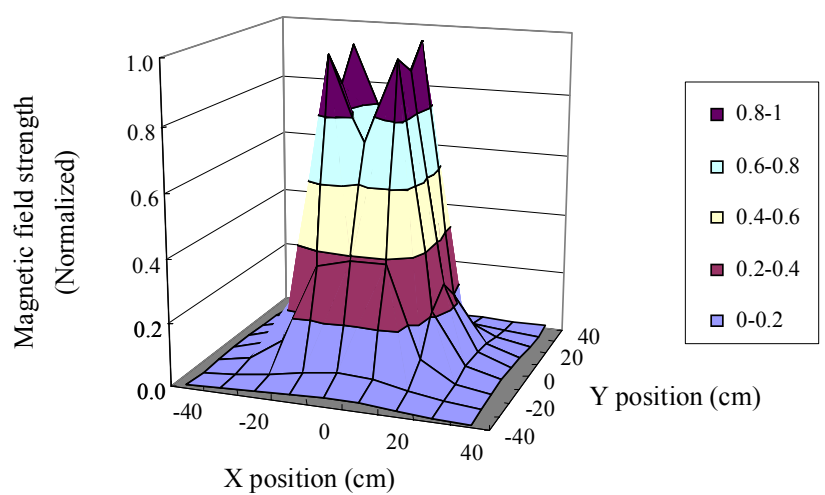

Figure 4. Magnetic field distributions form a HF band RFID reader/writer antenna. (Normalized to maximum magnetic field strength.)

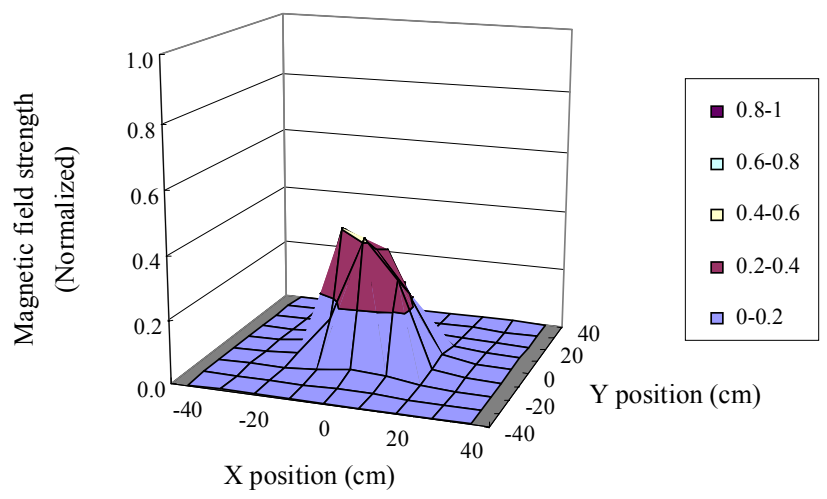

Figure 5. Magnetic field distributions form a HF band RFID reader/writer antenna. (Normalized to maximum magnetic field strength.)

average value of magnetic flux density through the area of the coil. Measured magnetic field distributions are used for this calculation.

2) This value is equivalent to the magnetic flux per unit area inside of the coil. This is expressed as follows:

$$
\phi_{a}=\sum_{S} B \cdot i_{n} / S
$$

where $\phi_{a}$ is magnetic flux per unit area, $B$ is measured magnetic flux density in each sample point and $S$ is the area of the coil.

3) Calculate induced voltage using Faraday's law of induction. Induced voltage is expressed as follows:

$$
V_{i}=-N \frac{d \phi_{a}}{d t}
$$

where $N=1$ for one turn coils. In addition, $d t$ is the same constant for all antennas, since the antenna speed is maintained during the experiments. Furthermore, assume all magnetic flux through the coil becomes 0 by the antenna motion.

4) This methodology cannot determine an absolute value of induced voltage as long as the impedance of the coil and mutual coupling coefficient between the coil and antenna are unknown. However, relative values of induced voltage compared with a given standard antenna can determine when 
the same configurations of the pacemakers and human torso phantom are employed for this EMI assessment.

5) Therefore, if there is an experimental result of EMI test for at least one antenna, EMI on specific pacemakers or ICDs can be assessed by measuring the magnetic field from the antenna.

Fig. 6 shows the estimated induced voltage based on magnetic flux distributions from each antenna. In addition, Fig. 7 and Fig. 8 show the induced voltages compared with

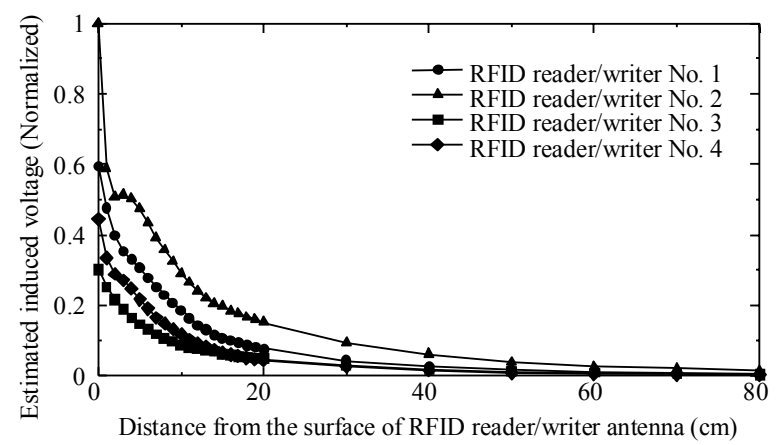

Figure 6. Estimated relative induced voltage (Normalized) by magnetic flux from $125 \mathrm{kHz}$ RFID reader/writer antennas.

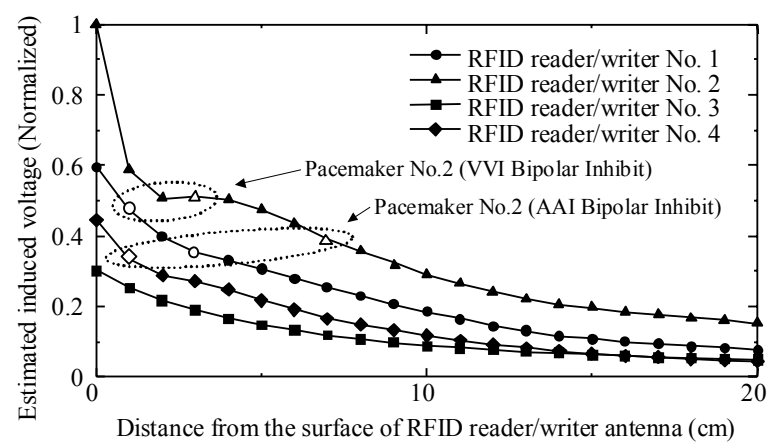

Figure 7. Estimated relative induced voltage (Normalized) by magnetic flux from $125 \mathrm{kHz}$ RFID reader/writer antennas compare with experimental results. (EMI test for a single chamber pacemaker.)

(Maximum interference distance is indicated as $\bigcirc \Delta \square \diamond$.)

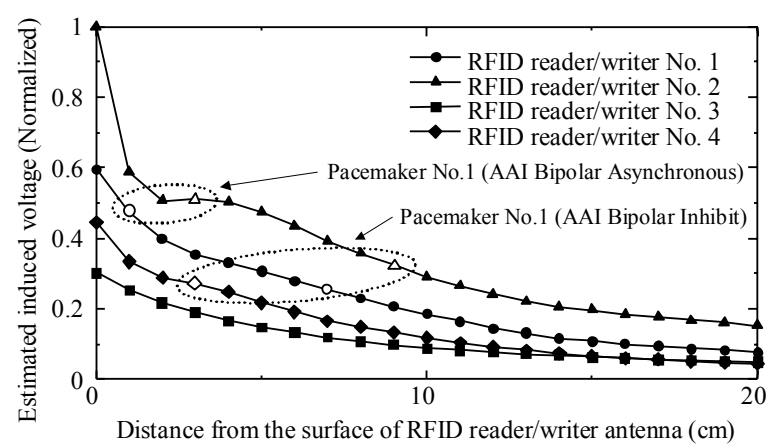

Figure 8. Estimated relative induced voltage (Normalized) by magnetic flux from $125 \mathrm{kHz}$ RFID reader/writer antennas compare with experimental results. (EMI test for a dual chamber pacemaker.) (Maximum interference distance is indicated as $\circ \Delta \square \diamond$.)

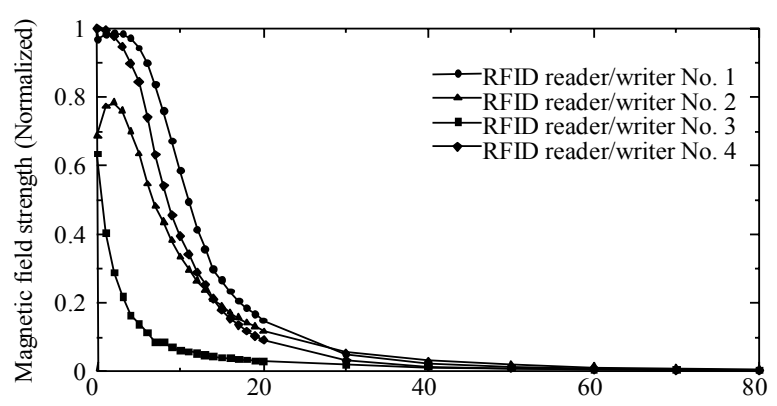

Distance from the surface of RFID reader/writer antenna $(\mathrm{cm})$

Figure 9. 1-dimensional magnetic field distributions of $125 \mathrm{KHz}$ RFID reader/writer antennas.

(Magnetic field strength on the axis of maximum strength.)

maximum interference distance obtained from the experiments. Maximum interference distances are plotted on the lines of induced voltage. Estimated induced voltages at each maximum interference distance are almost the same. These figures demonstrate results of estimation for 16 test modes and for 2 types of pacemakers. A total of 480 test modes are examined. Detailed evaluation of this methodology is required for statistical processing.

In addition, 1-dimensional magnetic field distributions of $125 \mathrm{kHz}$ RFID reader/writer antennas on the axis of maximum strength are shown in Fig. 9. Values of $y$-axis are normalized to the maximum field strength out of 4 types of antennas. From Fig. 6 compared with Fig. 9, RFID reader/writer No. 4 has the highest magnetic field strength at the surface of the antenna. Despite this, this antenna has a relatively low induced voltage and less EMI characteristics. By using this assessment methodology, predictions of antennas with less EMI characteristics may be possible.

\section{B. Statistical processing of the methodology results}

To confirm the effectiveness of the methodology, difference evaluations are carried out. Standard deviations of induced voltage for each pacemaker's test mode are calculated as shown in Fig. 10. The evaluations are conducted for the test modes which are affected by more than two antennas. The total number of test modes for the evaluations are 310 and 90 , respectively. Test modes which are not affected, or affected by only one antenna are excluded from the evaluations.

Fig. 11 shows the frequency table of the standard deviations calculated from the results of the methodology. In this case, it is supposed that an induced voltage can be derived from magnetic flux distributions. The $x$-axis shows standard deviation of the induced voltage for each test mode. In addition, the $y$-axis on the left hand side and right hand side shows frequency and cumulative ratio, respectively. The result shows that $80 \%$ of the cumulative ratio is within standard deviations of 0.1 .

Fig. 12 shows the frequency table of the standard deviations calculated from the maximum magnetic field strength. In this case, it is supposed that an induced voltage can be derived from maximum magnetic field strength. The result shows that more than $80 \%$ of the cumulative ratio is within standard deviations 
of 0.27 . However, standard deviations vary widely, and no trend is confirmed.

These results show that the induced voltages calculated from the proposed methodology have less uncertainty compared to those calculated from the maximum magnetic field strength. We can reconfirm that EMI due to low-band RFID reader/writer is affected by the magnetic flux distribution - not the maximum magnetic strength on the one point. In addition, by using the methodology, if there is an experimental result of an EMI test for at least one antenna, EMI on specific pacemakers or ICDs can be assessed by measuring the magnetic field from the antenna.

\section{CONCLUSION}

In this paper, EMI estimation methodology based upon the total magnetic flux integrated across the pacemaker and lead

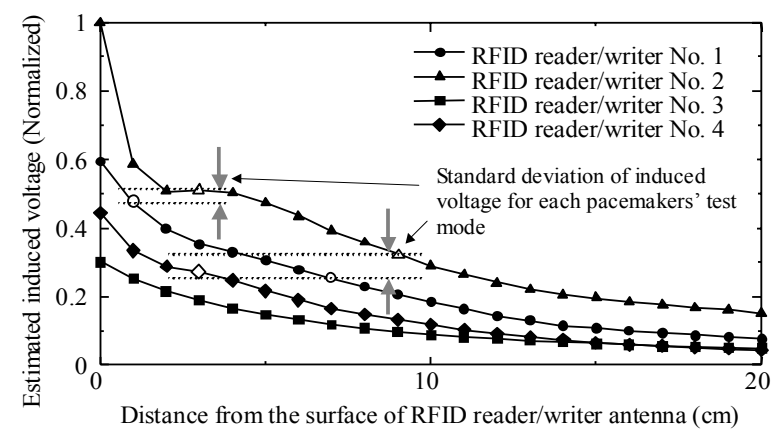

Figure 10. Difference evaluations of the metodlogy. (Maximum interference distance is indicated as $\bigcirc \Delta \square \diamond$.)

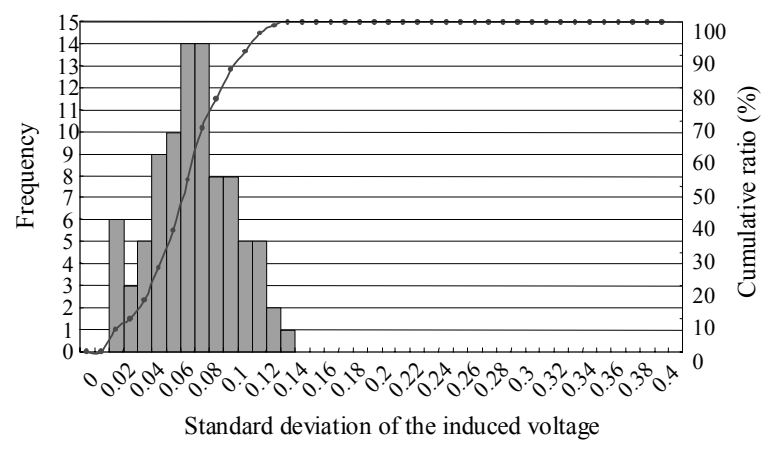

Figure 11. Standard deviations of the induced voltage calculated from the results of the methodology.

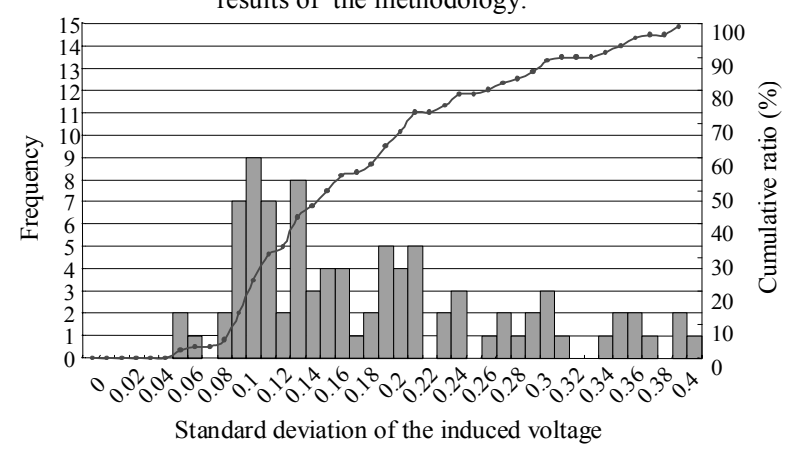

Figure 12. Standard deviations of the induced voltage calculated from the results of the maximum maghetic field strength. cross-section was compared with the experimental results to confirm the effectiveness of the proposed methodology. The induced voltages obtained by using the methodology were in good agreement with those obtained by the experiments. There is a possibility that EMI on pacemakers or ICDs can be assessed by measuring the magnetic field from the antenna considering the mutual coupling effects.

In the future, to achieve a more detailed EMI assessment, investigation of uncertainties of the proposed EMI estimation methodology will be carried out. In addition, to develop a computer simulation based on the EMI assessment method, numerical modeling of the EMI test system and the EMF analysis will also be carried out.

\section{ACKNOWLEDGEMENT}

The authors would like to thank the members of the Pacemaker Committee of Japan and Japan Automatic Identification Systems Association for their cooperation and support.

\section{REFERENCES}

[1] W. Irnich, L. Batz, R. Muller, and R. Tobisch, "Electromagnetic interference of pacemaker by mobile phones," PACE, vol. 19, pp.14311446, Oct. 1996.

[2] V. Barbaro, P. Bartolini, A. Donato, and C. Militello, "Electromagnetic interference of analog cellular telephone with pacemakers," PACE, vol. 19, pp. 1410-1418, Oct. 1996.

[3] T. Toyoshima, M. Tsumura, T. Nojima, and Y. Tarusawa, "Electromagnetic interference of implantable cardiac pacemakers by portable telephones," Japanese journal of cardiac pacing and electrophysiology, vol. 12, no.5, pp. 488-497, 1996.

[4] Y. Tarusawa, K. Ohshita, Y. Suzuki, T. Nojima, and T. Toyoshima "Experimental estimation of EMI from cellular base-station antennas on implantable cardiac pacemakers," IEEE Trans. Electromagn. Compat., vol. 47, no. 4, pp.938-950, Nov. 2005.

[5] T. Nojima, and Y. Tarusawa, "A new EMI test method for electronic medical devices exposed to mobile radio wave," IEICE trans. Commun., vol.E88-B, no.1, pp. 11-18, Jan. 2001

[6] V. Ivans, "Active implantable medical devices and electromagnetic compatibility", BIOLOGICAL EFFECTS of EMFs 4th International Workshop, Oct. 2006.

[7] "Investigation and research report of electromagnetic interference on medical devices (in Japanese)," Ministry of Internal Affairs and Communications (MIC) of Japan, Mar. 2005.

[8] "Guidelines on the use of radio communication equipment such as cellular telephones - Safeguards for electric medical equipment," presented at the EMC Conf. Japan, Electromagnetic Medical Equipment Study Group, 1997.

[9] "Guidelines to prevent interference on implantable medical devices from wireless devices," Ministry of Internal Affairs and Communications (MIC) of Japan, Aug. 2005.

[10] S. Futatsumori, T. Kono, T. Hikage, T. Nojima, and B. Koike, "Experimental test system to assess the EMI from RFID reader/writer on implantable cardiac pacemaker", in Proc. Progress in Electromagnetics Research Symposium (PIERS 2006), 2P3-5, p. 210, Aug. 2006.

[11] S. Futatsumori, T. Hikage, T. Nojima, B. Koike, H. Fujimoto and T Toyoshima, "In vitro experiments to assess electromagnetic fields exposure effects from RFID reader/writer for pacemaker patients," in Proc. BIOLOGICAL EFFECTS of EMFs 4th International Workshop, pp. 494-500, Oct. 2006.

[12] Active Implantable Medical Device - Electromagnetic Compatibility EMC Test Protocols for Implantable Cardiac Pacemakers and Implantable Defibrillators, AAMI Standard PC69, 1999. 